\title{
SELEÇÃO DE PLANTAS QUANTIFICADORAS DE HERBICIDAS RESIDUAIS
}

A determinação da concentração de compostos no solo por meio de plantas quantificadoras apresenta como principal vantagem detectar somente resíduos biologicamente ativos, não havendo necessidade de instrumentos onerosos e de prévia extração dos resíduos do solo. Dessa forma, este trabalho teve como objetivo selecionar plantas quantificadoras da presença de herbicidas residuais (pré emergentes) para o uso em bioensaios. Utilizou-se delineamento experimental completamente casualizado com arranjo bifatorial $8 \times 6$, com cinco repetições. $O$ fator A consistiu de espécies cultiváveis e o fator B de herbicidas aplicados em pré emergência. Os resultados evidenciaram que a sensibilidade na detecção do herbicida no solo depende da espécie utilizada. A sensibilidade das espécies Lactuca sativa L. e Raphanus sativus var. sativus L. não permitiu condições de quantificar a presença dos herbicidas atrazina, cloransulam, imazaquin, metribuzin e S-metolacloro. Raphanus sativus var. oleiferus Metzger é potencial quantificador de imazaquin e $\mathrm{S}$ metolacloro. Plantas de Curcubita pepo L. são promissoras na bioavaliação de metribuzin. A espécie Cucumis sativus L. mostrou-se potencial bioindicadora de cloransulan e imazaquin. Avena sativa L. apresentou-se como potencial quantificadora de imazaquin e metribuzin. Hordeum vulgare L. pode quantificar o metribuzin e Triticum aestivum L. é promissor na detecção da biodisponibilidade de atrazina.

PALAVRAS-CHAVE: BIOENSAIO; CINÉTICA DE DISSIPAÇÃO; MEIA-VIDA; HERBICIDAS.

* Engenheiro Agrônomo, Mestre em Fitotecnia, Bolsista CNPq, Doutorando em Fitotecnia, Universidade Federal do Rio Grande do Sul (UFRGS) Porto Alegre, RS (e-mail: nunes.ander@gmail.com).

** Engenheiro Agrônomo, PhD em Plantas Daninhas, Professor, Departamento Plantas de Lavoura, UFRGS, Porto Alegre, RS (e-mail: ribas.vidal@ufrgs.br). 


\section{INTRODUÇÃO}

A permanência do herbicida no solo, após a sua aplicação, pode determinar a eficácia no controle de plantas daninhas, sendo denominada período residual. Contrariamente, pode determinar problemas, tais como: danos à cultura subsequente e o risco de contaminação ambiental, sendo, então, denominada persistência (ROSS e LEMBI, 1999). O período de permanência varia com a adsorção, lixiviação, degradação e transformação química ou biológica e ainda mediante absorção e decomposição pelas plantas. Esses fatores regulam a concentração e o fluxo das moléculas desses compostos na solução do solo (LOUX, LIEBL e SLIFE, 1989; CELIS et al., 2006). Além disso, a importância e a intensidade da ação desses fatores sobre a persistência dependem das condições edafo-climáticas específicas dos locais de estudo (WALKER, MOON e WELCH, 1992; HOLLAWAY et al., 2006b).

Determina-se a permanência de compostos no solo mediante espectrometria de massa, cromatografia e pelo uso de plantas indicadoras (bioensaios). Os bioensaios apresentam como principal vantagem detectar somente resíduos biologicamente ativos, não havendo necessidade de instrumentos onerosos e de prévia extração dos resíduos do solo. Porém, os resultados apresentados são semiquantitativos e necessitam curvas de calibração para cada herbicida e para cada espécie indicadora (BEYER et al., 1988).

A partir da década de 30 , surgiram os primeiros ensaios utilizando plantas como indicadoras da presença de compostos no solo. Um dos primeiros ensaios realizados avaliou plantas do gênero Avena como indicadoras da presença de arsenito de sódio e cloreto de sódio em solos da Califórnia (CRAFTS, 1935). Essa técnica continua sendo muita utilizada em estudos de persistência, dissipação, lixiviação, atividade biológica e dose-resposta de herbicidas em solos (BRINTON, EVANS e BLEWETT, 2005; ZHOU e WANG, 2006; HOLLAWAY et al., 2006a; PANNACCI, ONOFRI e COVARELLI, 2006; SHANER et al., 2006; NUNES e VIDAL, 2008).

Cada herbicida, em virtude do tipo e número de átomos que o compõem e do seu arranjo estrutural, apresenta série específica de propriedades físico-químicas. 0 conhecimento das propriedades físico-químicas dos herbicidas assume grande importância, pois através delas é possível prever o comportamento dos mesmos no ambiente. A diversidade das propriedades físico-químicas torna difícil o uso de apenas uma espécie quantificadora em bioensaios com mais de um herbicida.

Para efeitos conceituais, considera-se planta quantificadora de determinado herbicida aquela que é medianamente sensível à presença desse herbicida e planta indicadora da presença de determinado herbicida aquela que apresenta qualquer nível de sensibilidade. Assim, nem toda planta indicadora pode ser quantificadora. Caso a sensibilidade da planta ao herbicida seja elevada, a planta responderá em faixa de dose muito limitada uma vez que as curvas de dose-resposta perdem a significância. Além disso, características importantes das espécies indicadoras incluem: alta taxa de crescimento, pois permitirá rápida visualização dos sintomas ocorridos; ampla distribuição geográfica para que as espécies possam ser utilizadas como indicadoras em outros locais de estudo; e homogeneidade genética, pois padroniza as respostas obtidas e possibilita maior homoscedasticidade da variância na análise dos dados. Normalmente, essas características são encontradas em espécies cultivadas, tendo em vista que os programas de melhoramento genético selecionam tais características. Dessa forma, este trabalho teve como objetivo selecionar plantas indicadoras da presença de herbicidas residuais (pré-emergentes) para o uso em bioensaios. 


\section{MATERIAL E MÉTODOS}

Conduziu-se o experimento entre junho e agosto de 2005, em casa de vegetação da Faculdade de Agronomia da Universidade Federal do Rio Grande do Sul (UFRGS), localizada no município de Porto Alegre. O solo utilizado nos vasos de $800 \mathrm{~cm}^{3}$, classificado como Argissolo Vermelho Distrófico típico (EMBRAPA, 1999), contém $18 \%$ de argila, $15 \mathrm{~g} \mathrm{~kg}^{-1} \mathrm{de}$ M.O., saturação de bases de $88 \%$, pH igual a 6,0, capacidade de troca de cátions a pH 7,0 de 15,7 cmol $\mathrm{kg}^{-1}$, fósforo superior a $100 \mathrm{mg} \mathrm{kg}^{-1}$, potássio superior a $400 \mathrm{mg} \mathrm{kg}^{-1}$.

Adotou-se delineamento experimental completamente casualizado com arranjo bifatorial $8 \times 6$ e cinco repetições (STEEL e TORRIE, 1980). O fator A consistiu das espécies cultiváveis: Avena sativa L. (AVESA), Cucumis sativus L. (CUMSA), Curcubita pepo L. (CUUPE), Hordeum vulgare L. (HORVX), Lactuca sativa L. (LACSA), Raphanus sativus var. oleiferus Metzger (RAPSO), Raphanus sativus var. sativus L. (RAPSR) e Triticum aestivum $L$. (TRZAX). O fator $B$ consistiu dos herbicidas de aplicação em pré emergência: atrazina (3000 $\left.\mathrm{g} \mathrm{ha}^{-1}\right)$, cloransulam (40 $\left.\mathrm{g} \mathrm{ha}^{-1}\right)$, imazaquin $\left(140 \mathrm{~g} \mathrm{ha}^{1}\right)$, metribuzin (1440 g ha $^{-1}$ ), S-metolacloro (1920 $\mathrm{g} \mathrm{ha}^{-1}$ ) e testemunha. Os herbicidas foram selecionados em função da sua importância agrícola e por apresentarem características físico-químicas contrastantes entre si.

Foram colocadas três sementes por vaso na profundidade de $1 \mathrm{~cm}$. Os herbicidas utilizados foram aspergidos ao solo úmido, duas vezes, uma no sentido contrário à outra, com pulverizador costal pressurizado com $\mathrm{CO}^{2}$ mantido à pressão constante de $200 \mathrm{kPa} e$ munido de bicos tipo leque 8001 , distanciados entre si em $0,50 \mathrm{~m}$ na barra de 1,5 $\mathrm{m}$ de largura, totalizando o volume de calda aspergida de $220 \mathrm{~L}$ ha-1. No momento da aplicação, a temperatura do ar variou de 21 a $23^{\circ} \mathrm{C}$ e a umidade relativa de 91 a 79\%. Após a aplicação dos herbicidas, realizou-se irrigação, simulando precipitação de $20 \mathrm{~mm}$. O desenvolvimento das plantas ocorreu em casa de vegetação com temperatura média de $22,3 \pm 8,2^{\circ} \mathrm{C}$ e umidade relativa do ar de $75,4 \pm 19,3 \%$, com irrigação por capilaridade.

As variáveis analisadas foram a fitotoxicidade dos herbicidas aos 14 dias após a aplicação (DAA), a estatura e a área foliar $\left(\mathrm{cm}^{2}\right)$ aos 21 DAA. Para a fitotoxicidade, atribuíramse notas que variaram de 0 para ausência de efeito a 100 para efeito letal nas plantas, incluindo a inibição da emergência por parte dos herbicidas. A estatura foi determinada mediante régua milimétrica. Determinou-se a área foliar com medidor de área foliar marca Li-cor, modelo 3100, com precisão de $0,01 \mathrm{~cm}^{2}$.

Os dados foram convertidos para porcentagem em relação à testemunha e transformados pela equação $\log (x+10)$, sendo em seguida submetidos à análise da variância pelo teste $F$. As variáveis analisadas foram avaliadas mediante comparação das médias de tratamentos pelo teste DMS a 5\% de probabilidade de erro experimental (STEEL e TORRIE, 1980).

\section{RESULTADOS E DISCUSSÃO}

A espécie LACSA apresentou elevada sensibilidade aos herbicidas utilizados (Tabela 1). No entanto, sua escolha como planta indicadora para quantificação é limitada porque na dose utilizada dos herbicidas houve elevada redução da estatura e área foliar (Tabelas 2 e 3 ). Isso indica a presença dos herbicidas no solo, mas dificulta a 
quantificação dos produtos em razão do elevado efeito nas plantas, não permitindo a análise quantitativa. Por se tratar de espécie muito sensível aos compostos químicos, seu uso está difundindo em testes de detecção de poluentes e aleloquímicos (USEPA, 1994). A espécie LACSA foi considerada apropriada para o uso em bioensaios de aleloquímicos, pois apresentou baixa variabilidade na germinação e desenvolvimento (MACIAS, CASTELLANO e MOLINILLO, 2000). Além disso, foi utilizada em bioensaio realizado para verificar a atividade biológica de compostos isolados de dois cultivares de Helianthus annuus (MACIAS et al., 2006). Em estudo de espécies indicadoras da presença de petróleo, somente a LACSA mostrou diferença significativa na germinação em resposta aos solos contaminado e não contaminado (BANKS e SCHULTZ, 2005).

\section{TABELA 1 - FITOTOXICIDADE ${ }^{1}$ DOS HERBICIDAS NAS OITO ESPÉCIES INDICADORAS AVALIADA AOS 14 DIAS APÓS A APLICAÇÃO (DAA)}

\begin{tabular}{|c|c|c|c|c|c|c|c|c|c|}
\hline \multirow{3}{*}{$\begin{array}{l}\text { Herbicida } \\
\text { Atrazina }\end{array}$} & \multirow{3}{*}{$\begin{array}{c}\text { Asteraceae } \\
\text { LACSA } \\
58 \mathrm{~B} \mathrm{a}\end{array}$} & \multirow{2}{*}{\multicolumn{2}{|c|}{$\frac{\text { Brassicaceae }}{\text { RAPSO RAPSR }}$}} & \multirow{2}{*}{\multicolumn{2}{|c|}{$\frac{\text { Cucurbitaceae }}{\text { CUUPE CUMSA }}$}} & \multicolumn{4}{|c|}{ Poaceae } \\
\hline & & & & & & \multicolumn{2}{|c|}{ AVESA } & \multirow{2}{*}{$\frac{\text { HORVX }}{\text { OC c }}$} & \multirow{2}{*}{$\frac{\text { TRZAX }}{9 \mathrm{Cc}}$} \\
\hline & & $58 \mathrm{~A} \mathrm{a}$ & $46 \mathrm{~A} a b$ & $50 \mathrm{~B} \mathrm{a}$ & $23 \mathrm{~B} \mathrm{~b}$ & $23 B$ & $\mathrm{~b}$ & & \\
\hline Cloransulam & $98 \mathrm{~A} a$ & $11 \mathrm{~B} \mathrm{~b}$ & $16 \mathrm{~B} \mathrm{~b}$ & $100 \mathrm{~A} a$ & $3 \mathrm{Cc}$ & $5 C D$ & c & $0 \mathrm{CC}$ & $19 \mathrm{~B} \mathrm{~b}$ \\
\hline Imazaquin & $58 \mathrm{~B} \quad \mathrm{a}$ & $56 \mathrm{~A} \mathrm{a}$ & $56 \mathrm{~A} \mathrm{a}$ & $43 \mathrm{~B}$ a & $1 \mathrm{Cd}$ & $16 \mathrm{BC}$ & C & $40 \mathrm{~B} a b$ & $20 B$ bc \\
\hline Metribuzin & $54 \mathrm{~B} a$ & $52 \mathrm{~A} \mathrm{a}$ & $52 \mathrm{~A} \mathrm{a}$ & $20 \mathrm{Cb}$ & $43 \mathrm{~A} \mathrm{a}$ & $38 \mathrm{~A}$ & $a b$ & $4 \mathrm{C} \mathrm{C}$ & $28 \mathrm{~B} \mathrm{~b}$ \\
\hline S-metolacloro & $100 \mathrm{~A} a$ & $62 \mathrm{~A} \mathrm{~b}$ & $52 \mathrm{~A} \mathrm{~b}$ & $46 \mathrm{~B} \mathrm{bc}$ & $52 \mathrm{~A} \mathrm{~b}$ & $21 B$ & c & $100 \mathrm{~A} \mathrm{a}$ & $100 \mathrm{~A} a$ \\
\hline Testemunha & $0 \mathrm{Ca}$ & $0 \mathrm{Ca}$ & $0 \mathrm{Ca}$ & $0 \mathrm{Da}$ & $0 \mathrm{Ca}$ & OD & a & $0 \mathrm{Ca}$ & $0 \mathrm{Ca}$ \\
\hline
\end{tabular}

${ }^{1}$ Escala de 0 a 100\%: $0 \%$ = sem fitotoxicidade, $100 \%$ = morte total.

LACSA = alface; RAPSO = nabo forrageiro; RAPSR = rabanete; CUUPE = abobrinha; CUMSA = pepino; $A V E S A=$ aveia branca; HORVX = cevada; TRZAX = trigo;

Médias seguidas pela mesma letra maiúscula nas colunas e minúscula nas linhas não diferem pelo teste DMS a 5\%.

Análise da variância com dados transformados pela equação log $(x+10)$; Quadrado Médio dos Herbicidas $=4,16$ ( $P>0,00001)$; Quadrado Médio das Espécies = 3,78 ( $>>0,00001)$; Quadrado Médio da Interação "Herbicidas*Espécies" $=1,99(P>0,00001) ;$ Quadrado Médio do Erro = 0,33; Coeficiente de Variação = 18,56\%.

Na família Brassicaceae, RAPSO apresentou-se como potencial indicadora e quantificadora da presença dos herbicidas imazaquin e S-metolacloro. Na presença desses produtos, a espécie mostrou média fitotoxicidade e redução intermediária na estatura e na área foliar (Tabelas 1 a 3). Isso permite elaborar curvas de dose-resposta para posterior quantificação desses herbicidas no solo. Cloransulan causou elevada redução na estatura e na área foliar das plantas, impossibilitando utilizar essas variáveis na sua quantificação. Plantas do gênero Raphanus foram testadas como indicadoras da presença de petróleo, mas as plantas produziram variabilidade na resposta (BANKS e SCHULTZ, 2005).

A espécie RAPSR demonstrou alta sensibilidade aos produtos utilizados, o que impossibilita sua utilização na quantificação dos produtos (Tabelas 1 a 3). O movimento e a persistência dos herbicidas imazaquin, oxifluorfem, flurocloridona e terbacil foram 
determinados pelo método de bioensaio baseado no desenvolvimento das plântulas dessa espécie (MILANOVA e GRIGOROV, 1996). Em bioensaio sob condições de laboratório, essa espécie foi utilizada com sucesso na avaliação de diferentes concentrações de óleo extraído de plantas de Eucalyptus citriodora (BATISH et al., 2004).

Na família Cucurbitaceae, CUUPE manifestou-se como potencial quantificadora da presença do herbicida metribuzin. Os produtos atrazina e imazaquin causaram elevada redução na estatura e área foliar da espécie, o que provavelmente dificultaria a utilização dessas variáveis na quantificação desses produtos no solo. O produto S metolacloro não causou efeito nas plântulas de CUUPE (Tabelas 1 a 3). Resíduos do herbicida imazapir, do mesmo grupo químico do imazaquin, não causaram efeitos em plantas de CUUPE (BOVEY e SENSEMAN, 1998). A espécie CUUPE foi utilizada em bioensaio, baseado no desenvolvimento radicular e de plântulas, realizado para verificar a atividade diferencial de compostos isolados de Secale cereale (BURGOS e TALBERT, 2000).

A espécie CUMSA apresentou-se como potencial quantificadora da presença dos produtos cloransulan e imazaquin, ambos herbicidas inibidores da enzima acetolactato sintase (ALS). Os demais herbicidas utilizados neste estudo reduziram a estatura e a área foliar, impossibilitando o emprego dessas variáveis na sua quantificação (Tabelas 2 e 3). Essa espécie foi utilizada sob condições de laboratório para investigar a persistência e a bioatividade do herbicida metsulfurom, também inibidor da enzima ALS (ISMAIL e AZLIZAN, 2002). A atividade herbicida de compostos derivados da fluorina foi estudada pelo método de bioensaio com plantas de CUMSA (LI et al., 2006). Outro bioensaio, baseado na germinação e desenvolvimento radicular de CUMSA, evidenciou a utilização da espécie como indicadora do efeito alelopático de extratos aquosos de plantas de Chelidonium majus (TAKACS, HORVATH e MIKULAS, 2004).

\section{TABELA 2 - ESTATURA EM RELAÇÃO À TESTEMUNHA DAS OITO ESPÉCIES INDICADORAS AVALIADAS AOS 21 DIAS APÓS A APLICAÇÃO (DAA)}

\begin{tabular}{|c|c|c|c|c|c|c|c|c|c|}
\hline \multirow{3}{*}{$\begin{array}{l}\text { Herbicida } \\
\text { Atrazina }\end{array}$} & \multirow{3}{*}{$\begin{array}{c}\text { Asteraceae } \\
\text { LACSA } \\
16 \text { B C }\end{array}$} & \multicolumn{2}{|c|}{ Brassicaceae } & \multicolumn{2}{|c|}{ Cucurbitaceae } & \multicolumn{4}{|c|}{ Poaceae } \\
\hline & & \multirow{2}{*}{$\begin{array}{l}\text { RAPSO } \\
21 \mathrm{C} \quad \mathrm{C}\end{array}$} & \multirow{2}{*}{$\begin{array}{c}\text { RAPSR } \\
0 \mathrm{Bd}\end{array}$} & \multirow{2}{*}{$\frac{\text { CUUPE }}{\text { OCd }}$} & \multirow{2}{*}{$\frac{\text { CUMSA }}{\text { O C d }}$} & \multirow{2}{*}{$\frac{\text { AVESA }}{2 \mathrm{Dd}}$} & \multirow{2}{*}{$\begin{array}{l}\text { HORVX } \\
97 \mathrm{~A} \mathrm{a}\end{array}$} & \multicolumn{2}{|c|}{ TRZAX } \\
\hline & & & & & & & & $41 \mathrm{~B}$ & $\mathrm{~b}$ \\
\hline Cloransulam & $19 \mathrm{~B}$ de & $11 \mathrm{C}$ de & $7 \mathrm{~B}$ ef & $0 \mathrm{Cf}$ & $43 \mathrm{~B} \mathrm{bc}$ & $99 \mathrm{~A} a$ & $76 \mathrm{~B} \mathrm{~b}$ & $25 \mathrm{BC}$ & $\mathrm{cd}$ \\
\hline Imazaquin & $19 \mathrm{~B} \mathrm{~b}$ & $45 B \quad a$ & $O B C$ & $6 \mathrm{C} \mathrm{bc}$ & $51 \mathrm{~B}$ a & $51 \mathrm{Ca}$ & $6 \mathrm{D} \mathrm{bc}$ & $6 \mathrm{D}$ & $b c$ \\
\hline Metribuzin & $19 \mathrm{~B} \mathrm{~b}$ & 1D c & $1 \mathrm{BC}$ & $48 \mathrm{~B} a$ & $0 \mathrm{Cc}$ & $41 \mathrm{Ca}$ & $34 \mathrm{C}$ ab & $18 \mathrm{~B}$ & $\mathrm{~b}$ \\
\hline S-metolacloro & $0 \mathrm{Cd}$ & $38 \mathrm{~B} \quad \mathrm{C}$ & $0 \mathrm{Bd}$ & $105 \mathrm{~A} \mathrm{a}$ & $0 \mathrm{Cd}$ & $73 \mathrm{~B} \mathrm{~b}$ & ODd & $O D$ & d \\
\hline Testemunha & $100 \mathrm{~A} a$ & $100 \mathrm{~A} a$ & $100 \mathrm{Aa}$ & $100 \mathrm{~A} a$ & $100 \mathrm{~A} \mathrm{a}$ & $100 \mathrm{~A} \mathrm{a}$ & $100 \mathrm{~A}$ a & $100 \mathrm{~A}$ & a \\
\hline
\end{tabular}

LACSA = alface; RAPSO = nabo forrageiro; RAPSR = rabanete; CUUPE = abobrinha; $C U M S A=$ pepino; $A V E S A=$ aveia branca; HORVX = cevada; TRZAX = trigo;

Médias seguidas pela mesma letra maiúscula nas colunas e minúscula nas linhas não diferem pelo teste DMS a 5\%. Análise da variância com dados transformados pela equação log $(x+10)$; Quadrado Médio dos Herbicidas $=16,36(P>$ 0,00001); Quadrado Médio das Espécies = 4,44 ( $>$ > 0,00001); Quadrado Médio da Interação "Herbicidas*Espécies" $=2,29$ $(P>0,00001)$; Quadrado Médio do Erro = 0,22; Coeficiente de Variação = 13,87\%. 
As espécies da família Poaceae apresentaram-se como potenciais quantificadoras de diversos produtos. Os herbicidas imazaquin e metribuzin podem ser potencialmente quantificados pela espécie AVESA. Para o herbicida S-metolacloro, essa espécie também se apresentou como potencial quantificadora, mas a fitotoxicidade e a redução de estatura não foram acentuadas, o que pode dificultar a quantificação do produto no solo por meio dessas variáveis (Tabelas 1 e 2). Essa espécie foi utilizada em bioensaios baseados no desenvolvimento das plântulas para o estudo da atividade, adsorção, mobilidade e persistência de diferentes formulações dos herbicidas alocloro, acetocloro, metolacloro, Smetolacloro, dimetenamida e flufenacet (VASILAKOGLOU e ELEFTHEROHORINOS, 1997; VASILAKOGLOU, ELEFTHEROHORINOS e DHIMA, 2001; VASILAKOGLOU e ELEFTHEROHORINOS, 2003).

O produto atrazina causou elevada fitotoxicidade e redução na estatura e na área foliar nas plantas de AVESA, indicando sua presença (Tabelas 1 a 3). AAVESA foi utilizada a campo e em laboratório para quantificação e estudo da eficiência e lixiviação dos produtos atrazina e simazina no solo (STORK, 1998). Além disso, essa espécie tem sido muito utilizada no estudo de solos contaminados e atividade de compostos alelopáticos. O nível de contaminação por metais pesados em diferentes solos foi avaliado pelo desenvolvimento e mudanças bioquímicas em plantas de AVESA (LOUREIRO et al., 2006). Já a influência alelopática das plantas de Conyza albida foi estudada por bioensaio baseado na germinação e desenvolvimento radicular de plantas de AVESA (ECONOMOU et al., 2002).

A espécie HORVX expressou-se como potencial quantificadora da presença do produto metribuzin. Os produtos imazaquin e S-metolacloro causaram inibição total na emergência da espécie HORVX. A espécie mostrou-se tolerante aos herbicidas atrazina e cloransulan (Tabelas 1 a 3). Entre diversas espécies estudadas, HORVX foi considerada apropriada para o uso em bioensaios com aleloquímicos por ter apresentado a mais baixa variabilidade na germinação e desenvolvimento (MACIAS, CASTELLANO e MOLINILLO, 2000). A influência alelopática de espécies do gênero Terminalia e a atividade biológica de flavonoides extraídos de diferentes cultivares de Helianthus annuus foram verificadas em ensaio com plantas de HORVX (BHATT, KUMAR e TODARIA, 1997; MACIAS et al., 1997). Essa espécie também foi utilizada em bioensaio para o estudo da atividade herbicida de auxinas sintéticas (ENGVILD, 1996).

A espécie TRZAX apresentou-se como potencial quantificadora da presença do herbicida atrazina e evidenciou média fitotoxicidade e redução intermediária na estatura e na área foliar em solo tratado com esse composto. O herbicida cloransulan reduziu a estatura e área foliar de TRZAX, impossibilitando utilizar essas variáveis na quantificação desse herbicida. Os herbicidas imazaquin e metribuzin causaram elevada fitotoxicidade e redução na estatura e área foliar de TRZAX. A espécie TRZAX foi considerada apropriada para o uso em bioensaios de aleloquímicos, pois apresentou baixa variabilidade na germinação e desenvolvimento (MACIAS, CASTELLANO e MOLINILLO, 2000). A persistência e meia-vida do herbicida clorsulfurom foram determinados por bioensaio com plantas de TRZAX (KINFE e PEEPER, 1993). A concentração dos produtos pendimetalina e flucloralina em solos foi determinada mediante bioensaio baseado no desenvolvimento radicular de plantas de TRZAX (DEV, SINGH e SINGH,1992). Além disso, bioensaio com essa espécie foi utilizado com sucesso na resposta a diferentes concentrações de óleo extraído de plantas de Eucalyptus citriodora (BATISH et al., 2004) e no estudo da atividade biológica de compostos isolados de dois cultivares de Helianthus annuus (MACIAS et al., 2006). Porém, entre diversas 
espécies testadas como indicadoras da presença de petróleo, TRZAX não mostrou diferença significativa na germinação em resposta ao solo contaminado e não contaminado, devido à alta variabilidade na resposta (BANKS e SCHULTZ, 2005).

\section{TABELA 3 - ÁREA FOLIAR EM RELAÇÃO À TESTEMUNHA DAS OITO ESPÉCIES INDICADORAS AVALIADAS AOS 21 DIAS APÓS A APLICAÇÃO (DAA)}

\begin{tabular}{|c|c|c|c|c|c|c|c|c|c|c|c|c|}
\hline \multirow{3}{*}{$\begin{array}{l}\text { Herbicida } \\
\text { Atrazina }\end{array}$} & \multirow{2}{*}{\multicolumn{2}{|c|}{$\frac{\text { Asteraceae }}{\text { LACSA }}$}} & \multicolumn{3}{|c|}{ Brassicaceae } & \multicolumn{2}{|c|}{ Cucurbitaceae } & \multicolumn{5}{|c|}{ Poaceae } \\
\hline & & & \multirow{2}{*}{$\begin{array}{l}\text { RAPSO } \\
1 \mathrm{C} \mathrm{C}\end{array}$} & \multicolumn{2}{|c|}{ RAPSR } & \multirow{2}{*}{$\begin{array}{c}\text { CUUPE } \\
O C \mathrm{C}\end{array}$} & \multirow{2}{*}{$\frac{\overline{C U M S A}}{0 \mathrm{CC}}$} & \multirow{2}{*}{$\begin{aligned} \text { AVESA } \\
5 \mathrm{C} \mathrm{C}\end{aligned}$} & \multicolumn{2}{|c|}{ HORVX } & \multicolumn{2}{|c|}{ TRZAX } \\
\hline & $5 B$ & c & & $O C$ & c & & & & $98 \mathrm{~A}$ & $\mathrm{a}$ & $38 \mathrm{~B}$ & $\mathrm{~b}$ \\
\hline Cloransulam & $1 B$ & d & $2 \mathrm{Cd}$ & $8 \mathrm{~B}$ & $\mathrm{~cd}$ & $0 \mathrm{Cd}$ & $41 \mathrm{~B} \mathrm{~b}$ & $98 \mathrm{~A}$ a & $98 \mathrm{~A}$ & $\mathrm{a}$ & $9 \mathrm{C}$ & c \\
\hline Imazaquin & $1 B$ & $\mathrm{~b}$ & $53 \mathrm{~B} \mathrm{a}$ & OC & $\mathrm{b}$ & $2 \mathrm{Cb}$ & $47 \mathrm{~B} \mathrm{a}$ & $63 \mathrm{~B} \mathrm{a}$ & $6 \mathrm{BC}$ & $\mathrm{b}$ & $3 \mathrm{CD}$ & $b$ \\
\hline Metribuzin & 1B & c & $1 \mathrm{C} \mathrm{C}$ & $3 \mathrm{BC}$ & $\mathrm{c}$ & $30 \mathrm{~B}$ a & $0 \mathrm{Cc}$ & $12 \mathrm{C} \mathrm{b}$ & $12 \mathrm{~B}$ & $\mathrm{~b}$ & $3 \mathrm{CD}$ & $c$ \\
\hline S-metol acloro & $O B$ & $d$ & $45 B C$ & OC & $d$ & $97 \mathrm{~A} \mathrm{a}$ & $0 \mathrm{Cd}$ & $68 \mathrm{~B} \mathrm{~b}$ & $O C$ & $d$ & $O D$ & $d$ \\
\hline Testemunha & $100 \mathrm{~A}$ & $\mathrm{a}$ & $100 \mathrm{~A} \mathrm{a}$ & $100 \mathrm{~A}$ & $\mathrm{a}$ & $100 \mathrm{~A} \mathrm{a}$ & $100 \mathrm{~A} \mathrm{a}$ & $100 \mathrm{~A} a$ & $100 \mathrm{~A}$ & $\mathrm{a}$ & $100 \mathrm{~A}$ & $\mathrm{a}$ \\
\hline
\end{tabular}

LACSA = alface; RAPSO = nabo forrageiro; RAPSR = rabanete; CUUPE = abobrinha $;$ CUMSA = pepino; AVESA = aveia branca; HORVX = cevada; TRZAX = trigo;

Médias seguidas pela mesma letra maiúscula nas colunas e minúscula nas linhas, não diferem pelo teste DMS a 5\%. Análise da variância com dados transformados pela equação log $(x+10)$; Quadrado Médio dos Herbicidas $=23,73(P>$ 0,00001); Quadrado Médio das Espécies = 5,04 (P > 0,00001); Quadrado Médio da Interação "Herbicidas*Espécies" = 2,34 $(P$ > 0,00001); Quadrado Médio do Erro = 0,09; Coeficiente de Variação = 9,72\%.

\subsection{CONSIDERAÇÕES NA ESCOLHA DA ESPÉCIE INDICADORA}

As espécies utilizadas apresentaram diferentes padrões de resposta aos herbicidas aspergidos no solo. A espécie potencialmente quantificadora da presença dos herbicidas utilizados no solo deve ser sensível para indicar a presença do produto, mas suficientemente tolerante para que possa desenvolver-se. Assim, mostrará os efeitos do herbicida e, consequentemente, apresentará potencial de resposta à dose. Espécies altamente sensíveis restringem a resposta dos herbicidas porque pequenas concentrações são suficientes para causar-Ihes elevados efeitos e, assim, a faixa de resposta à dose fica limitada. Da mesma forma, espécies tolerantes só apresentam efeitos sob elevada concentração do herbicida, restringindo a faixa de resposta em altas concentrações.

Considerando todos os resultados apresentados anteriormente, deparou-se com três situações distintas. A primeira quando a espécie não apresentou potencial quantificador dos herbicidas utilizados no solo, como RAPSR e LACSA, porque foram altamente sensíveis aos produtos. Assim, estão automaticamente excluídas da seleção de plantas quantificadoras por não atenderem ao objetivo inicial. Na segunda, a espécie apresentou potencial quantificador para apenas um dos produtos testados, como CUUPE e HORVX que poderiam ser utilizadas em bioensaios com metribuzin e TRZAX com atrazina. Na terceira situação, durante avaliação de bioensaios para permanência de mais de um herbicida, observou-se que uma espécie pode quantificar dois dos herbicidas utilizados, o que a torna mais 
interessante por exigir somente uma curva de dose-resposta, pois além de diminuir o volume de trabalho reduz também o erro experimental. Nesse caso, a espécie CUMSA pode quantificar os produtos cloransulam e imazaquin. Também existe a opção de utilizar a espécie RAPSO como quantificadora dos herbicidas imazaquin e S-metolacloro, produtos inibidores da enzima ALS e da parte aérea, respectivamente. A espécie AVESA pode ser empregada para os herbicidas imazaquin e metribuzin, inibidores da enzima ALS e fotossistema II, respectivamente.

\section{CONCLUSÃO}

A sensibilidade na detecção do herbicida depende da espécie utilizada. As espécies Lactuca sativa L. e Raphanus sativus var. sativus L. não evidenciaram potencial para quantificar os herbicidas atrazina, cloransulam, imazaquin, metribuzin e S-metolacloro. Raphanus sativus var. oleiferus Metzger é potencial quantificador de imazaquin e Smetolacloro. Plantas de Curcubita pepo L. são promissoras na bioavaliação de metribuzin. A espécie Cucumis sativus L. mostrou-se como potencial bioindicadora de cloransulan e imazaquin. Avena sativa L. é potencial quantificadora de imazaquin e metribuzin. Hordeum vulgare L. pode detectar o metribuzin e Triticum aestivum L. é promissor na detecção de atrazina.

\section{ABSTRACT}

\section{SELECTION OF RESIDUAL HERBICIDES QUANTIFYING PLANTS}

The determination of the concentration of compounds in the soil through plants quantifiers show as the main advantage to detect only biologically active residues, no need for expensive instruments and previous extraction of residues from the soil. Thus, this work aims to select indicator plants the presence of residual herbicides for use in bioassays. The experimental design used was the completely randomized, using the bifactorial arrangement $8 \times 6$, with five repetitions. The factor A consisted of the crops and factor B consisted of the preemergence herbicides application. The results show that the sensibility in the detection of herbicide in soil depends on the crop used. The sensibility of species Lactuca sativa L. and Raphanus sativus var. sativus L. don't permit conditions to quantify the presence of herbicides atrazine, cloransulam, imazaquin, metribuzin and S-metolachlor. Raphanus sativus var. oleiferus Metzger is potencial quantified of imazaquin and S-metolachlor. Plants of Curcubita pepo L. are promising in the bioevaluation of metribuzin. The specie Cucumis sativus L. is potential bioindicator of cloransulan and imazaquin. Avena sativa is potential to quantify imazaquin and metribuzin. Hordeum vulgare L. can quantify metribuzin and Triticum aestivum L. is promising in the detection of atrazine bioavailability

KEY-WORDS: BIOASSAY; DISSIPATION KINETICS; HALF-LIFE.

\section{REFERÊNCIAS}

1 BANKS, M. K.; SCHULTZ, K. E. Comparison of plants for germination toxicity tests in petroleumcontaminated soils. Water Air and Soil Pollution, Dordrecht, v.167, n.1-4, p. 211-219, 2005.

2 BATISH, D. R.; SETIA, N.; SINGH, H. P.; KOHLI, R. K. Phytotoxicity of lemon-scented eucalypt oil and its potential use as a bioherbicide. Crop Protection, Oxford, v.23, n.12, p.1209-1214, 2004.

3 BEYER, E. M.; DUFFY, M. J.; HAY, J. V.; SCHLUETER, D. D. Sulfunylureia. In: KEARNEY, P.C; KAUFMAN, D.D. (eds). Herbicides: chemistry, degradation, and mode of action. New York: M. Dekker, 1988. p.117189.

4 BHATT, B. P.; KUMAR, M.; TODARIA, N. P. Studies on the allelopathic effects of Terminalia species of Garhwal Himalaya. Journal of Sustainable Agriculture, Binghamton, v.11, n.1, p.71-84, 1997. 
5 BOVEY, R.W.; SENSEMAN, S.A. Response of food and forage crops to soil-applied imazapyr. Weed science, Lawrence, v.46, n.5, p.614-617, 1998.

6 BRINTON, W. F.; EVANS, E.; BLEWETT, T. C. Herbicide residues in composts: pH and salinity affect the growth of bioassay plants. Bulletin of Environmental Contamination and Toxicology, New York, v. 75, n. 5, p. 929936, 2005.

7 BURGOS, N. R.; TALBERT, R. E. Differential activity of allelochemicals from Secale cereale in seedling bioassays. Weed Science, Lawrence, n.48, n.3, p. 302-310, 2000.

8 CELIS, R.; REAL, M.; HERMOSIN, M.C.; CORNEJO, J. Desorption, persistence, and leaching of dibenzofuran in European soils. Soil Science Society of America Journal, Madison, v.70, n.4, p.13101317, 2006.

9 CRAFTS, A. S. The toxicity of sodium arsenite and sodium chlorate in four California soils. Hilgardia, Berkeley, v. 9, n. 4, p. 462-498, 1935.

10 DEV, J.; SINGH, J. N.; SINGH, G. Development and use of a soil bioassay for pendimethalin and fluchloralin. Tropical Pest Management, Basingstoke, v. 38, n.1, p. 22-24, 1992.

11 ECONOMOU, G.; TZAKOU, O.; GANI, A.; YANNITSAROS, A.; BILALIS, D. Allelopathic effect of Conyza albida on Avena sativa and Spirodela polyrhiza. Journal of Agronomy and Crop Science, Berlin, v.188, n. 4, p. 248-253, 2002.

12 EMBRAPA. Centro Nacional de Pesquisa de Solos. Sistema brasileiro de classificação de solos. Brasília, DF: Embrapa Produção de Informação; Rio de Janeiro: Embrapa Solos, 1999. 412 p.

13 ENGVILD, K.C. Herbicidal activity of 4-chloroindoleacetic acid and other auxins on pea, barley and mustard. Physiologia Plantarum, Oxford, v. 96, n. 2, p. 333-337, 1996.

14 HOLLAWAY, K. L.; KOOKANA, R. S.; NOY, D. M.; SMITH, J. G.; WILHELM, N. Persistence and leaching of imazethapyr and flumetsulam herbicides over a 4-year period in the highly alkaline soils of southeastern Australia. Australian Journal of Experimental Agriculture, Collingwood Victoria, v. 46, n. 5, p. 669-674, 2006a.

15 HOLLAWAY, K.L.; KOOKANA, R.S.; NOY, D.M.; SMITH, J.G.; WILHELM, N. Persistence and leaching of sulfonylurea herbicides over a 4-year period in the highly alkaline soils of south-eastern Australia. Australian Journal of Experimental Agriculture, Collingwood Victoria, v. 46, n. 8, p. 1069-1076, $2006 \mathrm{~b}$.

16 ISMAIL, B.S.; AZLIZAN, B.A. Persistence and bioactivity of metsulfuron-methyl in three soils. Journal of Environmental Science and Health - Part B - Pesticides Food Contaminants and Agricultural Wastes, New York, v. 37, n. 4, p. 345-353, 2002.

17 KINFE, B.; PEEPER, T.F. Persistence of chlorsulfuron and BAY SMY 1500 in air dry soil at roomtemperature. Weed Technology, Lawrence, v. 7, n.1, p. 29-32, 1993.

18 LI, G.Y.; QIAN, X.H.; CUI, J.N.; HUANG, Q.C.; CUI, D.W.; ZHANG, R.; LIU, F.Y. Synthesis and herbicidal activities of fluorine-containing 3-pyridylmethyl-2-phenyliminothiazolidine derivatives. Journal of Fluorine Chemistry, Amsterdam, v.127, n. 2, p.182-186, 2006.

19 LOUREIRO, S.; SANTOS, C.; PINTO, G.; COSTA, A.; MONTEIRO, M.; NOGUEIRA, A.J.A.; SOARES, A.M.V.M. Toxicity assessment of two soils from Jales mine (Portugal) using plants: growth and biochemical parameters. Archives of Environmental Contamination and Toxicology, New York, v. 50, n. 2, p.182-190, 2006.

20 LOUX, M.M.; LIEBL, R.A.; SLIFE, F.W. Availability and persistence of imazaquin, imazethapyr, and clomazone in soil. Weed Science, Champaign, v. 37, n.1, p. 259-267, 1989.

21 MACIAS, F.A.; CASTELLANO, D.; MOLINILLO, J.M.G. Search for a standard phytotoxic bioassay for allelochemicals. Selection of standard target species. Journal of Agricultural and Food Chemistry, Washington, v. 48, n. 6, p. 2512-2521, 2000.

22 MACIAS, F.A.; FERNANDEZ, A.; VARELA, R.M.; MOLINILLO, J.M.G.; TORRES, A.; ALVES, P.L.C.A. Sesquiterpene lactones as allelochemicals. Journal of Natural Products, Scottsdale, v.69, n. 5, p. 795800, 2006 
23 MACIAS, F.A.; MOLINILLO, J.M.G.; TORRES, A.; VARELA, R.M.; CASTELLANO, D. Bioactive flavonoids from Helianthus annuus cultivars. Phytochemistry, Oxford, v .45, n. 4, p.683-687, 1997.

24 MILANOVA, S.; GRIGOROV, P. Movement and persistence of imazaquin, oxyfluorfen, flurochloridone and terbacil in soil. Weed Research, Oxford, v.36, n.1, p.31-36, 1996.

25 NUNES, A.L.; VIDAL, R.A. Persistência do herbicida S-metolachlor associado ao glyphosate ou paraquat em plantio direto. Planta Daninha, Viçosa, v.26, n.2, p.385-393, 2008.

26 PANNACCI, E.; ONOFRI, A.; COVARELLI, G. Biological activity, availability and duration of phytotoxicity for imazamox in four different soils of central Italy. Weed Research, Oxford, v. 46, n. 3, p. 243-250, 2006.

27 ROSS, M.A.; LEMBI, C.A. Applied weed science. $2^{\text {nd }}$ ed. New Jersey: Prentice Hall, 1999. 452 p.

28 SHANER, D.L.; BRUNK, G.; BELLES, D.; WESTRA, P.; NISSEN, S.; WESTRA, P.; NISSEN, S. Soil dissipation and biological activity of metolachlor and S-metolachlor in five soils. Pest Management Science, Bognor Regis, v. 62, n. 7, p. 617-623, 2006.

29 STEEL, R.G.D.; TORRIE, J.H. Principles and procedures of statistics: a biometrical approach. $2^{\text {nd }}$ ed. New York: McGraw-Hill, 1980. 631 p.

30 STORK, P.R. Bioefficacy and leaching of controlled-release formulations of triazine herbicides. Weed Research, Oxford, v. 38, n. 6, p. 433-441, 1998.

31 TAKACS, A.P.; HORVATH, J.; MIKULAS, J. Inhibitory effect of Chelidonium majus extracts. Journal of Plant Diseases and Protection, Stuttgart, v.19, n.1, p. 285-292, 2004.

32 USEPA. United States Environmental Protection Agency. Using toxicity tests in ecological risk assessment. Eco Update, Washington, v. 2, n.1, p.1-12, 1994.

33 VASILAKOGLOU, I.B.; ELEFTHEROHORINOS, I.G. Activity, adsorption, mobility, efficacy, and persistence of alachlor as influenced by formulation. Weed Science, Lawrence, v. 45, n. 4, p. 579-585, 1997.

34 VASILAKOGLOU, I.B.; ELEFTHEROHORINOS, I.G.; DHIMA, K.B. Activity, adsorption and mobility of three acetanilide and two new amide herbicides. Weed Research, Oxford, v. 41, n. 6, p. 535-546, 2001.

35 VASILAKOGLOU, J.B.; ELEFTHEROHORINOS, I.G. Persistence, efficacy, and selectivity of amide herbicides in corn. Weed Technology, Lawrence, v.17, n.2, p.381-388, 2003.

36 WALKER, A.; MOON, Y.; WELCH, S.J. Influence of temperature, soil moisture, and soil characteristics on the persistence of alachlor. Pesticide Science, London, v. 35, n. 2, p.109-116, 1992.

37 ZHOU, X.H.; WANG, C.Y. Biological and biochemical detection techniques for glufosinate. Weed Science, Lawrence, v. 54, n. 3, p. 413-418, 2006. 